\title{
BMJ Open Awareness, usage and perceptions of authorship guidelines: an international survey of biomedical authors
}

\author{
Sara Schroter (D) , ${ }^{1}$ Ilaria Montagni (D) , ${ }^{2}$ Elizabeth Loder (D) , , ${ }^{1,3}$ M Eikermann (D) , ${ }^{4}$ \\ Elke Schäffner (D) , ${ }^{5}$ Tobias Kurth (1) ${ }^{5}$
}

To cite: Schroter S, Montagni I, Loder E, et al. Awareness, usage and perceptions of authorship guidelines: an international survey of biomedical authors. BMJ Open 2020;10:e036899. doi:10.1136/ bmjopen-2020-036899

- Prepublication history and additional material for this paper is available online. To view these files, please visit the journal online (http://dx.doi.org/10. 1136/bmjopen-2020-036899)

SS and IM contributed equally.

Received 10 January 2020 Revised 10 August 2020 Accepted 19 August 2020

\section{Check for updates}

(c) Author(s) (or their employer(s)) 2020. Re-use permitted under CC BY-NC. No commercial re-use. See rights and permissions. Published by BMJ.

${ }^{1}$ BMJ Publishing Group, London, UK

${ }^{2}$ Bordeaux Population Health Research Center UMR129, University of Bordeaux-Inserm, Bordeaux, France

${ }^{3}$ Division of Headache, Department of Neurology, Brigham and Women's Hospital, Boston, Massachusetts, USA ${ }^{4}$ Department of Anesthesia, Critical Care and Pain Medicine, Beth Israel Deaconess Medical Center, Boston, Massachusetts, USA

${ }^{5}$ Institute of Public Health, Charité - Universitätsmedizin Berlin, Berlin, Germany

Correspondence to

Dr llaria Montagni;

ilaria.montagni@u-bordeaux.fr

\section{ABSTRACT}

Objectives To investigate authors' awareness and use of authorship guidelines, and to assess their perceptions of the fairness of authorship decisions.

Design A cross-sectional online survey.

Setting and participants Corresponding authors of research papers submitted in 2014 to $18 \mathrm{BMJ}$ journals. Results 3859/12 646 (31\%) researchers responded. They worked in 93 countries and varied in research experience. Of these, 1326 (34\%) reported their institution had an authorship policy providing criteria for authorship; 2871 (74\%) were 'very familiar' with the International Committee of Medical Journal Editors' authorship criteria and 3358 $(87 \%)$ reported that guidelines were beneficial when preparing manuscripts. Furthermore, 2609 (68\%) reported that their use was 'sometimes' or 'frequently' encouraged in their research setting. However, 2859 respondents (74\%) reported that they had been involved in a study at least once where someone was added as an author who had not contributed substantially (honorary authorship), and 1305 (34\%) where someone was not listed as an author but had contributed substantially (ghost authorship) Only 740 (19\%) reported that they had never experienced either honorary or ghost authorship; 1115 (29\%) reported that they had experienced both at least once. There was no clear pattern in experience of authorship misappropriation by continent. For their last coauthored article, 2187 (57\%) reported that explicit authorship criteria had been used to determine eligibility, and 3088 (80\%) felt that the decision made was fair. When institutions frequently encouraged use of authorship guidelines, authorship eligibility was more likely to be discussed early (817 of $1410,58 \%)$ and perceived as fairer (1273 of $1410,90 \%$ ) compared with infrequent encouragement (974 of 2449, 40\%, and 1891 of $2449,74 \%$ ).

Conclusions Despite a high level of awareness of authorship guidelines and criteria, these are not so widely used; more explicit encouragement of their use by institutions may result in more favourable use of guidelines by authors.

\section{INTRODUCTION}

The research process, including publication, is based on trust. Authorship is both about being credited for the work you have done and being responsible and accountable for the integrity of what is published. ${ }^{1-3}$

\section{Strengths and limitations of this study}

- Very large international survey of active researchers describing their current practice.

- We address authorship practice, which is an important ethical matter because authorship ensures credit and accountability for research.

- We report self-administered survey data, and given the sensitivity of the questions, social desirability bias may have led respondents to over-report their awareness and usage of authorship guidelines.

Responsible authorship is a key component of publication ethics and transparent reporting. ${ }^{4}$ Infringing the rules of authorship in scientific papers can negatively impact on the credibility of the findings as well as on the honesty of the authors. However, the temptation for scientists to abuse authorship is significant since their publication record and collaboration with coauthors can determine academic rewards such as medical qualifications and professional appointments, as well as research funding. ${ }^{5}$ Studies reviewing published papers have identified a high prevalence of authorship problems. ${ }^{6-9}$

The average number of authors per published article has grown over time $\mathrm{e}^{1011}$ and this has raised questions around authorship in terms of eligibility, definition of their roles and establishment of a fair sequence of authors' names according to their role. ${ }^{12}$ Decisions about authorship eligibility can be subjective and contentious, since an author could contribute to the research without being involved in the actual writing, for example, by collecting data or conducting the statistical analysis. There is a huge variation in the operational definition of authorship 4 and preference for authorship order varies by country and discipline. ${ }^{12-14}$ In biomedicine, it is generally assumed that individuals are listed in decreasing order of level of their contribution with the exception of the last 
and the corresponding authors to whom importance is also attached. ${ }^{15}$ In other disciplines such as psychology, it is the first author who assumes responsibility for the publication and handles responses to inquiries after publication and coauthors are listed in order of level of contribution.

While authorship eligibility and order can reflect legitimate regional or discipline-specific practices, some scientists also intentionally misappropriate authorship. Honorary authors are those who did not contribute substantially to the conception or design of the work; or the acquisition, analysis or interpretation of the data for the work; or the writing of the article; and are unable to take public responsibility for the work. ${ }^{16}$ Honorary authorship may occur as a result of many factors, including nepotism; reciprocation of favours for previous authorships; institutional politics and power struggles; economic reasons to justify obtained grants or demands for new funding and trying to improve the chance of manuscript acceptance by including senior researchers. ${ }^{49}$ Ghost authors are those who are not listed as authors despite contributing substantially in these areas. ${ }^{16}$ Ghost authorship is especially undesirable when it masks the involvement of a commercial sponsor or other competing interests that could bias the study or reporting. ${ }^{17}$ Both honorary and ghost authorships are considered forms of research misconduct. Estimates from author surveys of the prevalence of honorary authors in high impact biomedical journals during the last 30 years have ranged from $19 \%$ to $39 \%^{71618}$ and ghost authors from $8 \%$ to $11 \%$. $^{71618}$

To help scientists define authorship and limit misconduct, multiple guidelines have been produced and journals have introduced various measures to try to encourage ethical authorship practice. $^{19}$ The International Committee of Medical Journal Editors (ICMJE) criteria, adopted by many international biomedical journals and generally considered the 'gold standard' for determining authorship eligibility, enumerate specific requirements for authorship, as well as stipulating that all authors should participate sufficiently in the work reported in an article to be able to take public responsibility for the content or an important part of the content ${ }^{20}$ While many journals continue to encourage the use of ICMJE criteria, previous studies of selected samples of researchers have shown poor awareness of them, ${ }^{21} 22$ dislike of them, ${ }^{22}{ }^{23}$ failure to comply with them ${ }^{6}$ and preference for other authorship policies and practices. ${ }^{24}$ One critic has even described them as illogical and unethical. ${ }^{25}$ Some journals have introduced their own authorship criteria. ${ }^{26}$ Others have shown that ICMJE criteria are intuitive and that the ICMJE-listed contributions are perceived as important. ${ }^{27}{ }^{28}$ However, there are no uniform rules for authorship order. ${ }^{14}$ We describe a large international survey undertaken to determine awareness and use of authorship guidelines and criteria in a contemporary sample of authors submitting papers to a broad range of biomedical journals.

\section{METHODS}

\section{Questionnaire development}

We developed a 12-item online closed questionnaire (see online supplemental appendix 1) with five additional demographic questions and a free-text item for additional comments. We piloted the questionnaire with 16 researchers to check for ambiguous items and revised the questionnaire in light of feedback. The final questionnaire included items addressing familiarity with and use of authorship criteria, experience of authorship misappropriation, frequency and timing of authorship discussions, perceived fairness of authorship decisions and institutional encouragement to use authorship criteria.

\section{Sample}

We included authors submitting research articles in 2014 to 18 journals covering a range of specialties published by BMJ Publishing Group (see online supplemental appendix 2). To try to get a broad sample of biomedical journals of varying size and prestige, we intentionally selected journals with high, middle and low impact factors (IFs). As a deviation from our protocol, we also sampled some recently acquired journals with no IF. All journals adhered to the ICMJE guidelines by asking corresponding authors to assure that they are respected.

\section{Procedures}

All corresponding authors of accepted and rejected research manuscripts submitted in 2014 were identified from each of the journal's manuscript tracking systems, and the data merged. Duplicate authors were removed so that each author was invited to take part in the survey only once. We selected one journal to act as a pilot to gauge response rate and invited eligible authors of this journal by an email on 14 March 2016 from SS, an employee of BMJ Publishing Group, to complete the survey hosted by SurveyMonkey; eligible authors of the other 17 journals were invited on 14 September 2016. Authors were informed that participation was voluntary and that responses would be anonymised and treated confidentially. Participants were not asked to give consent to take part; they were informed that completion of the survey would indicate that they had consented to take part. Non-responders were sent reminders at 2 weeks and 4 weeks after the initial mailing and the survey was open for completion for a 6-week period. To try to maximise recruitment, we gave an incentive of the chance to win a prize draw for a $£ 100$ voucher.

\section{Patient and public involvement}

We did not involve patients in the research team or development of the questionnaire as the focus was on academic researchers' perceptions and their institutional experiences. We recognise that patients are sometimes authors and may have different experiences as authors, but this forms only a small proportion of the published literature and patients' experience as authors was not the intended focus of the paper. To adequately capture patients' 
experience of authorship would require a different set of questions.

\section{Statistical analysis}

Responses from all journals were collated and the anonymised combined sample analysed using SPSS V.18. Quantitative data were summarised as frequencies and percentages.

\section{RESULTS}

\section{Sample}

Of the 12658 email invitations sent, 259 were not delivered by SurveyMonkey, 17 generated automated responses that recipients were on long-term leave or had retired and 3 recipients indicated they had been invited via a different email address. We received an actual response from 3859 (31\%) of the remaining 12379 authors. Response rates by journal ranged between $20 \%$ and $41 \%$. All results are presented as the number or proportion of all 3859 respondents unless explicitly stated otherwise.

Table 1 shows the characteristics of the sample. The majority of authors had submitted a paper that had been rejected by the sampled journal in 2014. A higher proportion of respondents were male $(56 \%)$ than female $(41 \%)$ and the majority $(71 \%)$ were based in a university setting. Respondents varied in research and publication experience and worked in 93 countries, with the highest proportions based in the UK (20\%), USA (10\%), Australia $(6 \%)$ and the Netherlands $(5 \%)$. Overall, the majority of respondents were based in Europe (54\%).

\section{Familiarity with and use of authorship criteria}

After being presented with the ICMJE criteria, 258 (7\%) reported that they had never heard of them, $706(18 \%)$ had heard of them but were not familiar with their content and $2871(74 \%)$ were very familiar with them. Of those who were very familiar with ICMJE criteria, $90 \%$ (2572/2871) reported that authorship guidelines and criteria were beneficial to research teams when preparing papers and deciding on authorship.

In relation to the last paper they coauthored, 2187/3859 $(57 \%)$ reported that explicit authorship criteria were used to decide who should be an author, 1284/3859 (33\%) said that they did not use them and 296/3859 (8\%) did not know. Only 1827 (64\%) of the 2871 who were very familiar with ICMJE criteria reported that explicit authorship criteria were used to decide who should be an author in their last coauthored paper.

\section{Authorship misappropriation}

Only around a quarter of researchers (929/3859) reported that they had never been involved in a study where someone was added as an author who did not contribute substantially (honorary authorship) (table 2). The frequency of involvement in studies with ghost authors was less than for honorary authors with nearly two-thirds of authors (2481/3859) never having been
Table 1 Respondent characteristics $(n=3859)$

n

$\%$

\begin{tabular}{lll}
\hline \multicolumn{2}{l}{ Editorial decision made on submitted article } & \\
Accept & 839 & 22 \\
Reject & 3020 & 78 \\
Gender & \\
Male & 2150 & 56 \\
Female & 1585 & 41 \\
Institution of work & & \\
University setting & 2739 & 71 \\
Public research centre & 511 & 13 \\
Private research centre & 113 & 3 \\
Industry & 29 & 1 \\
Other & 349 & 9
\end{tabular}

Number of years as an active researcher

\begin{tabular}{|c|c|c|}
\hline$<5$ years & 846 & 22 \\
\hline $6-10$ years & 1021 & 27 \\
\hline $11-15$ years & 628 & 16 \\
\hline 16-20 years & 462 & 12 \\
\hline More than 20 years & 772 & 20 \\
\hline \multicolumn{3}{|c|}{ Number of papers published } \\
\hline$\leq 5$ & 509 & 13 \\
\hline $6-10$ & 478 & 12 \\
\hline $11-20$ & 521 & 14 \\
\hline $21-30$ & 416 & 11 \\
\hline $31-40$ & 274 & 7 \\
\hline $41-50$ & 229 & 6 \\
\hline $51-100$ & 592 & 15 \\
\hline$>100$ & 689 & 18 \\
\hline \multicolumn{3}{|l|}{ Continent } \\
\hline Africa & 79 & 2 \\
\hline Asia & 652 & 17 \\
\hline Europe & 2073 & 54 \\
\hline North America & 594 & 15 \\
\hline South America & 90 & 2 \\
\hline Oceania & 243 & 6 \\
\hline
\end{tabular}

Percentages do not sum to $100 \%$ due to missing data.

involved in a study where someone was not listed as an author when they had contributed substantially.

Only around a fifth of all respondents $(740 / 3859,19 \%)$ reported that they had never experienced either guest or ghost authorship, whereas nearly a third (1115/3859, $29 \%$ ) reported that they had experienced both at least once in their careers. Researchers who had been active for more than 10 years reported a higher frequency of experience of authorship misappropriation than those who had been active for less than 10 years. Respondents who reported their institution had an authorship policy 
Table 2 Experience of authorship misappropriation by years of research experience

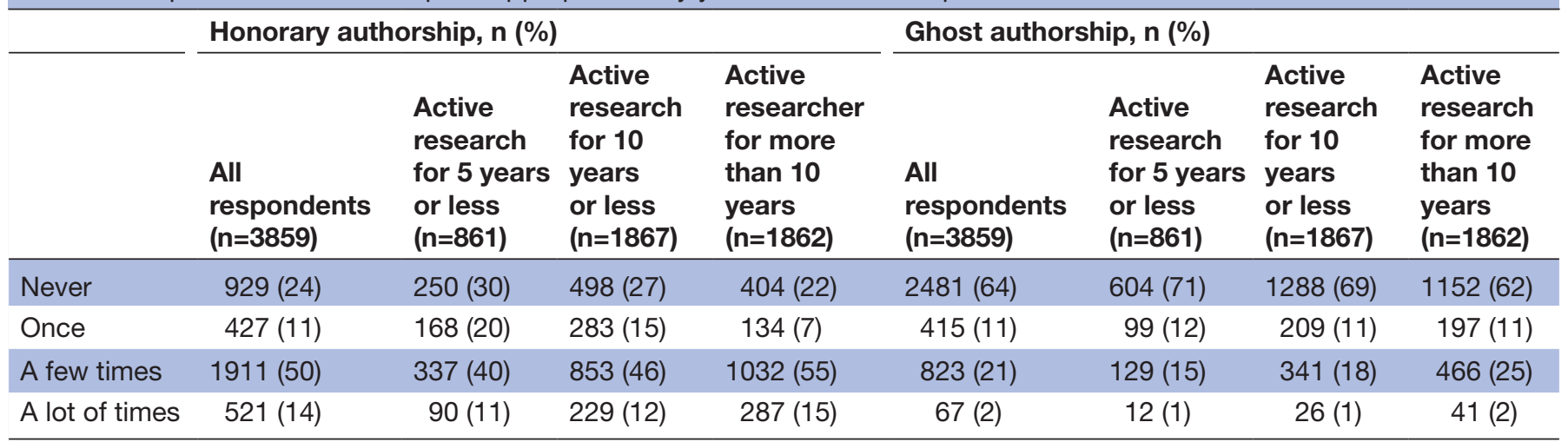

Percentages do not sum to $100 \%$ due to missing data.

were more likely $(374 / 1326,28 \%)$ to have never been involved in a study with honorary authorship than those who reported their institution did not have an authorship policy $(301 / 1592,19 \%)$. We found no clear pattern of perceived authorship misappropriation by continent (see online supplemental appendix 3).

\section{Timing of authorship discussions}

Authorship eligibility and authorship order were discussed at an early stage more often when institutions had authorship policies, when authors were very familiar with ICMJE criteria, when institutions encouraged use of authorship guidelines frequently and when explicit authorship criteria were used to decide who should be an author, compared with not (see online supplemental appendices 4 and 5).

Authorship eligibility was discussed at both an early stage and during the course of the study for a small proportion of recently coauthored articles and authorship order was discussed at both these points even less frequently, even when authorship institutional policies were in place.

\section{Perceived fairness of authorship decisions}

In relation to the last paper they coauthored, $80 \%$ (3088/3859) of respondents felt that the decision on who was made an author was fair (486, $13 \%$ not fair) and $82 \%$ (3157) felt that the decision on authorship order was fair (409, $11 \%$ not fair). When explicit criteria were used in authorship decisions, a higher proportion reported that the decision made on authorship eligibility (2043/2187, 93\%) and authorship order (2015/2187, 92\%) was fair, compared with when they were not used $(879 / 1284,69 \%)$ and $(946 / 1284,74 \%)$, respectively, as shown in online supplemental appendix 6. More experienced researchers and those working in settings where the use of criteria was actively encouraged reported higher rates of fairness for authorship decisions on their last coauthored paper than less experienced researchers and those working in settings where the use of criteria was not actively encouraged.
Institutional policy

Only 34\% (1326/3859) of respondents reported that their institution had an authorship policy; 41\% (1592) said that there was no such policy and 24\% (919) said that they did not know. For institutions with an authorship policy, 724/1326 (55\%) frequently encouraged researchers to use it and 434/1326 (33\%) sometimes.

Overall, when institutions frequently encouraged the use of authorship guidelines, decisions were more likely to be discussed at an early stage, were perceived as fairer and incidences of honorary and ghost authorships were reported as less common compared with when frequent institutional encouragement was not reported (infrequent, no encouragement, not sure and other) (table 3).

\section{DISCUSSION}

Our large survey of nearly 4000 active researchers from 93 countries found that almost three-quarters were very familiar with the ICMJE authorship criteria and a higher proportion viewed these and other authorship guidelines as beneficial. Around two-thirds reported that their institution frequently or sometimes encouraged the use of these or similar authorship criteria. Yet, only just over half used explicit authorship criteria when deciding on authorship for their last coauthored paper. When institutions frequently encouraged the use of authorship guidelines, authorship eligibility was more likely to be discussed early and was perceived as fairer. Reported incidences of authorship misappropriation over the course of researchers' careers were high; around three-quarters of respondents had experienced honorary authorship and one-third ghost authorship. Respondents self-reported multiple barriers to using authorship criteria in practice.

\section{Comparison with other studies}

Our results build on the results of earlier surveys ${ }^{7} 1618$ by providing a snapshot of authorship practice from a very large international sample of active researchers in a broad range of biomedical specialties. Similar to previous studies, ${ }^{7} 16$ we found reported rates of honorary 
Table 3 Stratification of responses by whether the use of explicit authorship guidelines and criteria in current research setting is frequently encouraged or not

\begin{tabular}{|c|c|c|}
\hline & \multicolumn{2}{|l|}{ n (\%) } \\
\hline & $\begin{array}{l}\text { Use of explicit } \\
\text { authorship } \\
\text { guidelines frequently } \\
\text { encouraged ( } n=1410)\end{array}$ & $\begin{array}{l}\text { Use of explicit } \\
\text { authorship guidelines } \\
\text { not frequently } \\
\text { encouraged }(n=2404)^{\star}\end{array}$ \\
\hline $\begin{array}{l}\text { Agrees that the explicit use of authorship guidelines and criteria is beneficial } \\
\text { to research teams when preparing a paper and deciding on authorship }\end{array}$ & $1330(94)$ & $2025(84)$ \\
\hline $\begin{array}{l}\text { Never been involved in a study where someone was not listed as an author } \\
\text { when they contributed substantially (ghost authorship) }\end{array}$ & $951(67)$ & $1526(64)$ \\
\hline Never experienced honorary or ghost authorship & $350(25)$ & $388(16)$ \\
\hline Experienced both honorary and ghost authorships & $370(26)$ & $744(31)$ \\
\hline $\begin{array}{l}\text { Felt decision on who should be an author on their last coauthored paper was } \\
\text { a fair reflection of who did what }\end{array}$ & $1273(90)$ & $1810(75)$ \\
\hline $\begin{array}{l}\text { Felt decision on order of authorship on their last coauthored paper was a fair } \\
\text { reflection of who did what }\end{array}$ & $1266(90)$ & $1886(79)$ \\
\hline
\end{tabular}

*Includes responses of 'other', 'not sure', 'not encouraged' and 'sometimes encouraged'.

authorship were higher than for ghost authorship. The proportion who had experienced honorary and ghost authorships was higher than previous surveys conducted between 1998 and 2011, ${ }^{718}$ but our respondents were asked about experience across their careers rather than about a specific publication and we did not just include high impact journals. We found no clear pattern of perceived authorship misappropriation by continent, which is in contrast to the findings of a systematic review in 2011, which found authorship problems and misuse were reported more often by researchers outside of the USA and the UK. ${ }^{9}$

Researchers in our study reported a higher level of familiarity and use of authorship guidelines and criteria than previous studies. ${ }^{21} 22$ This may partly be explained by wider promotion of these criteria and changes in authorship practice over time. For example, in some Nordic countries, compulsory courses on authorship guidelines have been introduced from the first year of the $\mathrm{PhD}$ programme. Early researchers are trained to discuss with their supervisor how to establish an equitable authorship order for papers. We may also have observed a higher level of familiarity and use of authorship guidelines because our sample was larger and composed of corresponding authors of articles submitted to journals promoting ICMJE criteria and requesting compliance with these criteria prior to publication.

However, despite such familiarity, more than one-third of our sample declared that explicit authorship criteria were not used to decide who should be an author on their most recent article. Similarly, Bonekamp et $a l^{24}$ found a high rate of awareness $(81 \%)$ of ICMJE criteria among submitting authors, yet $25 \%$ reported that at least one of their coauthors on the submission did not merit authorship. Our respondents described the difficulties of applying authorship criteria when, for example, colleagues disregard them, there are power imbalances and a strong cultural norm to attribute authorship in certain ways. Research culture is increasingly characterised by unhealthy competition, job insecurity, poor supervision and mentorship, discrimination, bullying and harassment, ${ }^{29}$ which can only have a negative impact on the quality of research and compliance with authorship guidelines and criteria. Early career researchers in particular can be pressured by supervisors to produce more research papers in journals with high IFs. The inclusion of senior researchers as coauthors, irrespective of their contribution, can increase the chances of publication in a competitive field. In addition, honorary authorship can give coauthors opportunities to strengthen collaborations with other researchers and increase the visibility of their work.

\section{Study limitations}

Our study has several limitations. First, we received a low $(31 \%)$ response rate, which may have caused selection bias. However, response rates to surveys of doctors and researchers are often low. ${ }^{30-33}$ Only a fifth of invited authors had their papers accepted by the journals in the sampling period and this may have affected their willingness to help. Also, some authors informed us that they only received 
the last reminder email, suggesting that some institutional email filters were treating the emails as spam. Despite the low response rate, we did receive nearly 4000 responses from all continents, which is a substantial survey sample.

Second, however, by surveying submitting authors and not just those who had papers accepted for publication at the participating journals, we sought to capture the experience of researchers from numerous countries and of varying levels of research and publication experience; some respondents will have never published with BMJ Publishing Group before. We also sampled authors submitting to a range of journals in different specialties and with a range of IFs.

Third, analyses are based on self-reported data from corresponding authors. We assured participants of confidentiality, but the survey was not anonymous and given the sensitivity of the questions, we cannot rule out social desirability bias with respondents over-reporting their awareness and usage of authorship criteria. We chose to contact corresponding authors as they coordinate the activities of other authors and are the people most likely to have knowledge of the roles and contributions of other authors. ${ }^{16}$

Finally, respondents completed the survey in 2016 and as such responses might not accurately reflect the current research ecosystem, which is continuously evolving in terms of publication policies and strategies.

\section{Study implications}

Understanding authorship practice is an important ethical matter because appropriate authorship ensures credit and accountability for research. Ethical authorship practice is essential for the promotion and maintenance of the scientific integrity of biomedical research. We found that authorship guidelines and criteria are known by the majority of researchers and their application is considered beneficial when preparing manuscripts. However, authorship misconduct is still prevalent; even those who are new to research reported experience of it. Thus, it is not simply a matter of authors needing to be informed about guidelines and criteria, but of having the opportunity to apply them in a supportive environment that is suited to their discipline.

While both institutions and journals have important duties relating to authorship misconduct, ${ }^{19}$ institutions are ultimately responsible for the conduct of their researchers. ${ }^{34}$ In 2000, a Task Force on Authorship reporting to the Council of Science Editors stated that all universities, medical schools, research institutes and commercial companies that conduct and publish research should have explicit policies on authorship. ${ }^{13}$ Yet 16 years later, only a third of respondents reported that their institution had an authorship policy, although this might partly be explained by researchers being unaware of existing institutional policies and the need for better promotion of these. Where institutions encouraged the use of authorship guidelines and criteria, perceptions of fairness of authorship decisions were higher and discussions on authorship eligibility and authorship order were more frequent. Little guidance exists on authorship order, which remains one of the major issues for most institutions. Institutions should be more active in supporting the use of authorship guidelines and criteria, especially to support early career researchers and to reduce power differentials among authorship teams. ${ }^{15}$ Authorship eligibility was discussed at an early stage and during the study for only a small proportion of recently coauthored articles in our sample. Proponents of good authorship practice recommend early discussion of authorship in the research process, ${ }^{35}$ something that could easily be encouraged by institutions. While it might be ideal but not feasible to have universal criteria for how researchers are recognised in publications, having well-designed institutional systems for agreeing and enforcing local and specific authorship policies at the start of projects and throughout the research process could help in avoiding disputes or resolving them quickly. ${ }^{15}$

On the other side, editors and publishers of some biomedical journals are already encouraging the use of authorship guidelines. In some journals, when submitting manuscripts, authors must indicate explicitly that all authors meet the journal's criteria for authorship, some even request completion of individual authorship confirmation forms. Other journals indicate in their instructions to authors that papers must meet authorship criteria, but do not explicitly enforce this and leave the responsibility of respecting these criteria to the authors. Recognising the ICMJE criteria may be unworkable in practice, some journals have preferred to introduce their own criteria for authorship. For example, Neurology recently revised its authorship policy, to recognise an author as someone who has substantially contributed to one or more of the following: design or conceptualisation of the study; major role in the acquisition of data; analysis or interpretation of the data or drafting or revising the manuscript for intellectual content. ${ }^{26}$ ICMJE requires authors to fulfil all four of its criteria, whereas Neurology requires just one of its criteria to be met.

In 1997, recognising the need for systemic reform, Rennie $e t a l^{2}$ proposed the introduction of published contributorship statements whereby individuals are named against their specific contributions and individuals can be mentioned without being authors on the byline, but most journals have not adopted this approach. This is also not accepted in most promotion committees for academic awards, where the authorship position counts. However, CRediT (Contributor Roles Taxonomy) ${ }^{36}$ has more recently been widely adopted by a range of publishers. CRediT has 14 different roles within the taxonomy and its approach is a step towards more transparency in the definition of coauthors since the roles of each author need to be recognised, categorised and listed when submitting to a journal. However, many argue that journal policies around authorship criteria lead to a meaningless tick-box exercise and studies have shown that published contributions often do not meet ICMJE criteria. ${ }^{8}$ Much of the science is based on trust and journal editors should not 
adjudicate authorship disputes or police authorship practice, but they should provide clear advice to authors and reviewers and have appropriate policies for editors and staff relating to all aspects of publication ethics. ${ }^{34}$ Journals should stipulate that authorship is about accountability as well as credit and authorship misappropriation is considered a form of research misconduct.

While courses in research ethics are now more common, many research institutions do not teach courses on publication ethics and only a small minority of international researchers report having substantial knowledge of publication ethics. ${ }^{37}$ The Committee on Publication Ethics (COPE) was set up to educate and support editors and publishers and those involved in publication ethics to foster good ethical practice in scientific publication. It provides, among others, guidelines to ensure that authorship and contributorship are in place, as well as clear policies that allow for transparency around who contributed to the work and in what capacity. While its members are mainly editors and publishers, COPE recently launched a new initiative to work in collaboration with several research institutions in Australia, Canada and the USA to help address issues around publication ethics commonly seen in journals further upstream (https://publicationethics.org/news/ cope-pilot-initiative-institutional-membership). Dealing with transgressions in publication ethics at the time of publication is often too late, so embedding good research practice within research institutions is crucial.

Modifying the 'microsystem' of authorship in biomedical research is a challenge that needs to be promptly addressed. Some argue that institutions, journal editors and funding agencies could introduce more stringent policies and punishments around authorship misappropriation. ${ }^{19}$ But it is the research culture that we need to change individual researchers' perceptions of moral behaviour. Guidelines cannot ensure morally responsible research, especially when they are limited to a checklist-like approach instead of an 'abstraction' level. ${ }^{38}$ The existence of these guidelines can paradoxically lead to a vision of researchers as people to distrust since they need a jurisdictional framework to practice their profession. Authorship guidelines and criteria should not be considered as merely strict rules to be respected in a normative way, but a ground for discussion about ethical choices and responsibilities of individual authors.

Despite a high level of awareness of authorship guidelines and criteria, these are not so widely used. More explicit encouragement by institutions to discuss authorship early and frequently may result in decisions that are perceived as fairer.

Contributors TK had the idea for the study (conception and design). IM reviewed the literature. SS and IM wrote the first draft of the manuscript and are joint first authors of this paper. SS managed the survey, collected and analysed the data, had full access to all the data and can take responsibility for the integrity of the data and the accuracy of the data analysis. All co-authors, including ME, EL and ES, participated in the design of the survey, interpretation of the results, revising the manuscript, and review and approval of the final manuscript. All authors meet the ICMJE authorship criteria and authorship eligibility.
Funding The authors have not declared a specific grant for this research from any funding agency in the public, commercial or not-for-profit sectors.

Competing interests All authors have completed the Unified Competing Interest form at www.icmje.org/coi_disclosure.pdf (available on request from the corresponding author) and declare that SS is a full-time employee at the BMJ. TK reports having contributed to an advisory board of CoLucid and a research project funded by Amgen, for which the Charité - Universitätsmedizin Berlin received an unrestricted compensation. TK further reports having received honoraria from Lilly, Newsenselab and Total for providing methodological advice, from Novartis and Daiichi Sankyo for providing a lecture on neuroepidemiology and research methods, and from the BMJ for editorial services. EL receives salary from the BMJ for services as head of research, paid to her employer, the Brigham and Women's Physician Organisation. IM reports having worked as an independent medical writer for Novartis, Sanofi SA and Bristol Myers Squibb. ES has received honoraria from Fresenius Medical Care, Fresenius Kabi and Siemens Healthineers for lectures.

Patient consent for publication Not required.

Ethics approval The study protocol was reviewed by the BMJ's ethics committee (7 0ctober 2015) and it did not have any major ethical concerns. Participation in the survey was voluntary and participants were told that they could withdraw at any stage. Participants were assured that the survey was confidential. The data were managed in compliance with the EU General Data Protection Regulation (GDPR).

Provenance and peer review Not commissioned; externally peer reviewed.

Data availability statement Data are available upon reasonable request. Anonymised individual respondent data will be shared on reasonable request.

Open access This is an open access article distributed in accordance with the Creative Commons Attribution Non Commercial (CC BY-NC 4.0) license, which permits others to distribute, remix, adapt, build upon this work non-commercially, and license their derivative works on different terms, provided the original work is properly cited, appropriate credit is given, any changes made indicated, and the use is non-commercial. See: http://creativecommons.org/licenses/by-nc/4.0/.

\section{ORCID iDs}

Sara Schroter http://orcid.org/0000-0002-8791-8564

llaria Montagni http://orcid.org/0000-0003-0076-0010

Elizabeth Loder http://orcid.org/0000-0003-1501-2947

M Eikermann http://orcid.org/0000-0002-7893-0596

Elke Schäffner http://orcid.org/0000-0002-7925-4577

Tobias Kurth http://orcid.org/0000-0001-7169-2620

\section{REFERENCES}

1 Rennie D, Flanagin A. Guests, ghosts, grafters, and the two-sided coin. JAMA 1994;271:469-71.

2 Rennie D, Yank V, Emanuel L, et al. A proposal to make contributors accountable. JAMA 1997;278:579-85.

3 Stephenson J. ICMJE: All authors of medical journal articles have "responsibility to stand by the integrity of the entire work". JAMA 2013;310:1216.

4 Sheikh A. Publication ethics and the research assessment exercise: reflections on the troubled question of authorship. $J$ Med Ethics 2000;26:422-6.

5 Wager E, Singhvi S, Kleinert S. Too much of a good thing? an observational study of prolific authors. PeerJ 2015;3:e1154.

6 upak-Smolčić V, Mlinarić A, Antončić D, et al. ICMJE authorship criteria are not Met in a substantial proportion of manuscripts submitted to Biochemia MEDICA. Biochem Med 2015;25:324-34.

7 Flanagin A, Carey LA, Fontanarosa PB, et al. Prevalence of articles with honorary authors and ghost authors in peer-reviewed medical journals. JAMA 1998;280:222-4.

8 Bates T, Anić A, Marusić M, et al. Authorship criteria and disclosure of contributions: comparison of 3 general medical journals with different author contribution forms. JAMA 2004;292:86-8.

9 Marušić A, Bošnjak L, Jerončić A. A systematic review of research on the meaning, ethics and practices of authorship across scholarly disciplines. PLoS One 2011;6:e23477.

10 Weeks WB, Wallace AE, Kimberly BCS. Changes in authorship patterns in prestigious US medical journals. Soc Sci Med 2004;59:1949-54.

11 US National Library of Medicine. Number of authors per MEDLINE/ PubMed citation. Available: https://www.nlm.nih.gov/bsd/authors1. html [Accessed 10 Dec 2019]. 
12 Tscharntke T, Hochberg ME, Rand TA, et al. Author sequence and credit for contributions in multiauthored publications. PLOS Biol 2007;5:e18.

13 Davidoff F. Who's the author? Problems with biomedical authorship, and some possible solutions (science editor). , 2000: 23, 111-9.

14 Perneger TV, Poncet A, Carpentier M, et al. Thinker, Soldier, Scribe: cross-sectional study of researchers' roles and author order in the Annals of Internal Medicine. BMJ Open 2017;7:e013898.

15 Wager E. Recognition, reward and responsibility: why the authorship of scientific papers matters. Maturitas 2009;62:109-12.

16 Wislar JS, Flanagin A, Fontanarosa PB, et al. Honorary and ghost authorship in high impact biomedical journals: a cross sectional survey. BMJ 2011;343:d6128.

17 Wager E, Field EA, Grossman L. Good publication practice for pharmaceutical companies. Curr Med Res Opin 2003;19:149-54.

18 Mowatt G, Shirran L, Grimshaw JM, et al. Prevalence of honorary and ghost authorship in Cochrane reviews. JAMA 2002;287:2769-71.

19 Bavdekar SB. Authorship issues. Lung India 2012;29:76-80.

20 International Committee of Medical Journal Editors. Defining the role of authors and contributors. Available: http://www.icmje.org/ recommendations/browse/roles-and-responsibilities/defining-therole-of-authors-and-contributors.html [Accessed 02 Jun 2020].

21 Bhopal R, Rankin J, McColl E, et al. The vexed question of authorship: views of researchers in a British medical faculty. BMJ 1997;314:1009-12.

22 Pignatelli B, Maisonneuve $\mathrm{H}$, Chapuis F. Authorship ignorance: views of researchers in French clinical settings. J Med Ethics 2005;31:578-81.

23 Hoen WP, Walvoort HC, Overbeke AJ. What are the factors determining authorship and the order of the authors' names? A study among authors of the Nederlands Tijdschrift voor Geneeskunde (Dutch journal of medicine). JAMA 1998;280:217-8.

24 Bonekamp S, Halappa VG, Corona-Villalobos CP, et al. Prevalence of honorary coauthorship in the American journal of roentgenology. AJR Am J Roentgenol 2012;198:1247-55.

25 Shaw D. The ICMJE's definition of authorship is illogical and unethical. BMJ 2011;343:d7192.
26 Amato AA, Baskin PK, Gross RA. Updating neurology authorship criteria: Ensuring inclusion of those making valuable intellectual contributions. Neurology 2018;90:865.

27 Ivaniš A, Hren D, Marušić M, et al. Less work, less respect: authors' perceived importance of research contributions and their declared contributions to research articles. PLoS One 2011;6:e20206-e.

28 Hren D, Sambunjak D, Ivanis A, et al. Perceptions of authorship criteria: effects of student instruction and scientific experience. $J$ Med Ethics 2007;33:428-32.

29 Wellcome. What researchers think about the culture they work in, 2020. Available: https://wellcome.ac.uk/reports/what-researchersthink-about-research-culture [Accessed 02 Jun 2020].

30 Cunningham CT, Quan H, Hemmelgarn B, et al. Exploring physician specialist response rates to web-based surveys. BMC Med Res Methodol 2015;15:32.

31 Kellerman SE, Herold J. Physician response to surveys. A review of the literature. Am J Prev Med 2001;20:61-7.

32 Mulligan A, Hall L, Raphael E. Peer review in a changing world: an international study measuring the attitudes of researchers. J Am Soc InformSci Tech 2013;64:132-61.

33 Price A, Schroter S, Clarke M, et al. Role of supplementary material in biomedical journal articles: surveys of authors, reviewers and readers. BMJ Open 2018;8:e021753.

34 Wager E, Kleinert S, Committee on Publication Ethics (COPE) Cooperation between research institutions and journals on research integrity cases: quidance from the Committee on publication ethics (COPE). Maturitas 2012;72:165-9.

35 Albert T, Wager E. How to handle authorship disputes: a guide for new researchers (the cope report 2003), 2003.

36 Allen L, O'Connell A, Kiermer V. How can we ensure visibility and diversity in research contributions? how the contributor role taxonomy (credit) is helping the shift from authorship to contributorship. Learn Pub 2019;32:71-4.

37 Schroter S, Roberts J, Loder E, et al. Biomedical authors awareness of publication ethics: an international survey. BMJ Open 2018;8:e021282

38 Johnsson L, Eriksson S, Helgesson G, et al. Making researchers moral: why trustworthiness requires more than ethics guidelines and review. Res Ethics 2014:10:29-46. 